\title{
RESEARCH NOTES
}

\section{A NEW PROOF OF MONOTONICITY FOR EXTENDED MEAN VALUES}

\author{
FENG QI, SEN-LIN XU, and LOKENATH DEBNATH
}

(Received 1 January 1997 and in revised form 5 July 1998)

ABSTRACT. In this article, a new proof of monotonicity for extended mean values is given.

Keywords and phrases. Monotonicity, extended mean values, integral form, arithmetic mean, Tchebycheff's integral inequality.

1991 Mathematics Subject Classification. Primary 26A48; Secondary 26D15.

1. Introduction. Stolarsky [14] first defined the extended mean values $E(r, s ; x, y)$ and proved that it is continuous on the domain $\{(r, s ; x, y): r, s \in R, x, y>0\}$ as follows

$$
\begin{aligned}
& E(r, s ; x, y)=\left(\frac{r}{s} \cdot \frac{y^{s}-x^{s}}{y^{r}-x^{r}}\right)^{1 /(s-r)}, \quad r s(r-s)(x-y) \neq 0 ; \\
& E(r, 0 ; x, y)=\left(\frac{y^{r}-x^{r}}{\ln y-\ln x} \cdot \frac{1}{r}\right)^{1 / r}, \quad r(x-y) \neq 0 ; \\
& E(r, r ; x, y)=e^{-1 / r}\left(\frac{x^{x^{r}}}{y^{y^{r}}}\right)^{1 /\left(x^{r}-y^{r}\right)}, \quad r(x-y) \neq 0 ; \\
& E(0,0 ; x, y)=\sqrt{x y}, \quad x \neq y ; \\
& E(r, s ; x, x)=x, \quad x=y .
\end{aligned}
$$

It is convenient to write $E(r, s ; x, y)=E(r, s)=E(x, y)=E$.

Several authors including Leach and Sholander [2, 3], Páles [6] and Yao and Cao [15] studied the basic properties, monotonicity and comparability of the mean values $E$. Feng Qi [9] and in collaboration with Qiu-mig Luo [7] further investigated monotonicity of $E$ from new viewpoints. Recently, Feng Qi [7] generalized the extended mean values and the weighted mean values $[1,4,5]$ as a new concept of generalized weighted mean values with two parameters, and studied its monotonicity and other properties.

In this note, a new proof of monotonicity for extended mean values is given.

2. Lemmas. Let

$$
\begin{aligned}
g=g(t)-g(t ; x, y) & =y^{t}-x^{t} / t, t \neq 0 ; \\
g(0 ; x, y) & =\ln y-\ln x .
\end{aligned}
$$


It is easy to see that $g$ can be expressed in integral form as

$$
g(t ; x, y)=\int_{x}^{y} u^{t-1} d u, \quad t \in R
$$

and

$$
g^{(n)}(t)=\int_{x}^{y}(\ln u)^{n} u^{t-1} d u, \quad t \in R
$$

Therefore, the extended mean values can be represented in terms of $g$ by

$$
\begin{aligned}
& E(r, s ; x, y)=\left(\frac{g(s ; x, y)}{g(r ; x, y)}\right)^{1 /(s-r)}, \quad(r-s)(x-y) \neq 0 ; \\
& E(r, r ; x, y)=\exp \left(\frac{g_{r}^{\prime}(r ; x, y)}{g(r ; x, y)}\right), \quad x-y \neq 0 .
\end{aligned}
$$

Set $F=F(r, s)=F(x, y)=F(r, s ; x, y)=\ln E(r, s ; x, y)$, then $F$ also can be expressed as

$$
\begin{aligned}
& F(r, s ; x, y)=\frac{1}{s-r} \int_{r}^{s} \frac{g_{t}^{\prime}(t ; x, y)}{g(t ; x, y)} d t, \quad r-s \neq 0 ; \\
& F(r, r ; x, y)=\frac{g_{r}^{\prime}(r ; x, y)}{g(r ; x, y)} .
\end{aligned}
$$

LEMmA 2.1. Assume that the derivative $f^{\prime \prime}(t)$ exists on an interval I. If $f(t)$ is an increasing or convex downward function respectively on $I$, then the arithmetic mean of $f(t)$,

$$
\begin{aligned}
& \phi(r, s)=\frac{1}{s-r} \int_{r}^{s} f(t) d t, \\
& \phi(r, r)=f(r),
\end{aligned}
$$

is also increasing or convex downward respectively with $r$ and $s$ on $I$.

Proof. Direct calculation yields

$$
\begin{aligned}
\frac{\partial \phi(r, s)}{\partial s} & =\frac{1}{(s-r)^{2}}\left[(s-r) f(s)-\int_{r}^{s} f(t) d t\right], \\
\frac{\partial^{2} \phi(r, s)}{\partial s^{2}} & =\frac{(s-r)^{2} f^{\prime}(s)-2(s-r) f(s)+2 \int_{r}^{s} f(t) d t}{(s-r)^{3}} \equiv \frac{\varphi(r, s)}{(s-r)^{3}}, \\
\frac{\partial \varphi(r, s)}{\partial s} & =(s-r)^{2} f^{\prime \prime}(s) .
\end{aligned}
$$

In the case of $f^{\prime}(t) \geq 0, \partial \phi(r, s) / \partial s \geq 0$, thus, $\phi(r, s)$ increases with $r$ and $s$, since $\phi(r, s)=\phi(s, r)$.

In the case of $f^{\prime \prime}(t) \geq 0, \varphi(r, s)$ increases with $s$. Since $\varphi(r, r)=0$, it is easy to see that $\partial^{2} \phi(r, s) / \partial s^{2} \geq 0$ holds. Therefore, $\phi(r, s)$ is convex downward with respect to either $r$ or $s$, since $\phi(r, s)=\phi(s, r)$. 
LEMMA 2.2. Let $f, h:[a, b] \rightarrow R$ be integrable functions, both increasing or both decreasing. Furthermore, let $p:[a, b] \rightarrow R$ be an integrable and nonnegative function. Then

$$
\int_{a}^{b} p(u) f(u) d u \int_{a}^{b} p(u) h(u) d u \leq \int_{a}^{b} p(u) d u \int_{a}^{b} p(u) f(u) h(u) d u .
$$

If one of the functions of $f$ or $h$ is nonincreasing and the other nondecreasing, then the inequality in (2.8) is reversed.

The inequality (2.8) is called Tchebycheff's integral inequality; for details, see [1, 4].

LEMMA 2.3. Let $i, j, k \in N$, we have

$$
g^{(2(i+k)+1)}(t ; x, y) g^{(2(j+k)+1)}(t ; x, y) \leq g^{(2 k)}(t ; x, y) g^{(2(i+j+k+1))}(t ; x, y) .
$$

If $x, y \geq 1$, then

$$
g^{(i+k)}(t ; x, y) g^{(j+k)}(t ; x, y) \leq g^{(k)}(t ; x, y) g^{(i+j+k)}(t ; x, y)
$$

If $0<x, y \leq 1$, then

$$
\begin{aligned}
g^{(2 i+k+1)}(t ; x, y) g^{(2 j+k+1)}(t ; x, y) & \leq g^{(k)}(t ; x, y) g^{(2(i+j+1)+k)}(t ; x, y) \\
g^{(2 i+k+1)}(t ; x, y) g^{(2 j+k)}(t ; x, y) & \geq g^{(k)}(t ; x, y) g^{(2(i+j)+k+1)}(t ; x, y) \\
g^{(2 i+k)}(t ; x, y) g^{(2 j+k)}(t ; x, y) & \leq g^{(k)}(t ; x, y) g^{(2(i+j)+k)}(t ; x, y) .
\end{aligned}
$$

Proof. By Tchebycheff's integral inequality (2.8) applied to the functions $p(u)=$ $(\ln u)^{2 k} u^{t-1}, f(u)=(\ln u)^{2 i+1}$ and $h(u)=(\ln u)^{2 j+1}$ for $i, j, k \in N, u \in[x, y], t \in R$, inequality (2.9) follows easily.

By the same arguments, inequalities (2.10), (2.11), (2.12), and (2.13) also follow from Tchebycheff's integral inequality.

LEMMA 2.4. The functions $g_{t}^{(2(k+i)+1)}(t ; x, y) / g_{t}^{(2 k)}(t ; x, y)$ are increasing with respect to $t, x$, and $y$ for $i$ and $k$ being nonnegative integers.

Proof. By simple computation, we have

$$
\left(\frac{g^{(2(k+i)+1)}(t)}{g^{(2 k)}(t)}\right)^{\prime}=\frac{g^{(2(i+k+1))}(t) g^{(2 k)}(t)-g^{(2(i+k)+1)}(t) g^{(2 k+1)}(t)}{\left[g^{(2 k)}(t)\right]^{2}} .
$$

Combining (2.9) and (2.14), we conclude that the derivative of $g^{(2(k+i)+1)}(t) / g^{(2 k)}(t)$ with respect to $t$ is nonnegative, and $g^{(2(k+i)+1)}(t ; x, y) / g_{t}^{(2 k)}(t ; x, y)$ increases with $t$. Differentiating directly, using the integral expression (2.3) of $g$ and rearranging gives 


$$
\begin{aligned}
\frac{\partial}{\partial y} & \left(\frac{g_{t}^{(2(k+i)+1)}(t ; x, y)}{g_{t}^{(2 k)}(t ; x, y)}\right) \\
= & \frac{\partial / \partial y\left[g_{t}^{(2(k+i)+1)}(t ; x, y)\right] g_{t}^{(2 k)}(t ; x, y)-g_{t}^{(2(k+i)+1)}(t ; x, y) \partial / \partial y\left[g_{t}^{(2 k)}(t ; x, y)\right]}{\left[g_{t}^{(2 k)}(t ; x, y)\right]^{2}} \\
= & \frac{y^{t-1}(\ln y)^{2 k}}{\left[g_{t}^{(2 k)}(t ; x, y)\right]^{2}}\left[(\ln y)^{2 i+1} \int_{x}^{y}(\ln u)^{2 k} u^{t-1} d u-\int_{x}^{y}(\ln u)^{2(i+k)+1} u^{t-1} d u\right] \geq 0 .
\end{aligned}
$$

Therefore, the desired monotonicity with respect to both $x$ and $y$ follows, for the involved functions are symmetric in $x$ and $y$. This completes the proof.

\section{Proof of monotonicity}

THEOREM 3.1. The extended mean values $E(r, s ; x, y)$ are increasing with respect to both $r$ and $s$ or to both $x$ and $y$.

Proof. This is a simple consequence of Lemma 2.1 and Lemma 2.3 in combination with its integral forms (2.4) and (2.5) of $E(r, s ; x, y)$.

REMARK 1. It may be pointed out that the method used in this paper could yield more general results (see [4, 12], and so on).

ACKNOWLEDGEMENT. The first author was partially supported by NSF grant 974050400 of Henan Province, China.

\section{REFERENCES}

[1] J.-C. Kuang, Changyong budengshi. [Applied inequalities], 2nd ed., Hunan Jiaoyu Chubanshe, Changsha, China, 1993 (Chinese). MR 95j:26001.

[2] E. B. Leach and M. C. Sholander, Extended mean values, Amer. Math. Monthly 85 (1978), 84-90. MR 58 22428. Zbl 379.26012.

[3] _ Extended mean values II, J. Math. Anal. Appl. 92 (1983), 207-223. MR 85b:26007. Zbl 517.26007.

[4] D. S. Mitrinović, Analytic Inequalities, Springer-Verlag, Berlin, 1970. MR 43\#448. Zbl 199.38101.

[5] D. S. Mitrinović, J. E. Pečaric, and A. M. Fink, Classical and New Inequalities in Analysis, Kluwer Academic Publishers, 1993. MR 94c:00004. Zbl 771.26009.

[6] Z. Páles, Inequalities for differences of powers, J. Math. Anal. Appl. 131 (1988), 271-281. MR 89f:26023. Zbl 649.26014.

[7] F. Qi, Generalized weighted mean values with two parameters, Proc. R. Soc. Lond., Ser. A, Math. Phys. Eng. Sci. 454 (1998), no. 1978, 2723-2732. Zbl 990.09932.

[8]___ On a two-parameter family of nonhomogeneous mean values, Tamkang Journal of Mathematics 8 (1998), no. 2.

[9] F. Qi and Q.-M. Luo, Refinement and extensions of an inequality, Mathematics and Informatics Quarterly (1998), to appear.

[10]__ A simple proof of monotonicity for extended mean values, J. Math. Anal. Appl. 224 (1999), no. 2, 356-359. Zbl 980.55030.

[11] F. Qi and S.-L. Xu, A new proof of monotonicity for generalized weighted mean values, submitted. 
[12] _ Refinements and extensions of an inequality. II, J. Math. Anal. Appl. 211 (1997), 616-620. CMP 9715.

[13] _ The function $\left(b^{x}-a^{x}\right) / x$ : inequalities and properties, Proc. Amer. Math. Soc. 126 (1998), no. 11, 3355-3359. CMP 9715.

[14] K. B. Stolarsky, Generalizations of the logarithmic mean, Math. Mag. 48 (1975), 87-92. MR 50 10186. Zbl 302.26003.

[15] R. Yang and D. Cao, Generalizations of the logarithmic mean, J. Ningbo Univ. (1989), no. 2, 105-108.

QI: DePARTMENT of MAThematics, Jiaozuo Institute of TeChnology, Jiaozuo City, HENAN 454000, CHINA

Xu: Department of MAthematics, University of Science AND TeChNOlogy of ChinA, HeFEI City, ANHui 230026, CHINA

DEBNATH: DEPARTMENT OF MATHEMATICS, UNIVERSITY OF CENTRAL FLORIDA, ORLANDO, FL 32816, USA 


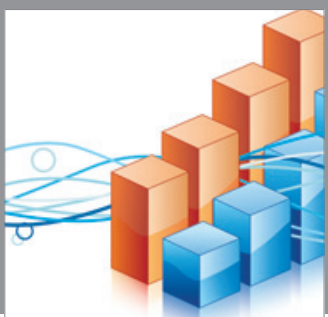

Advances in

Operations Research

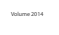

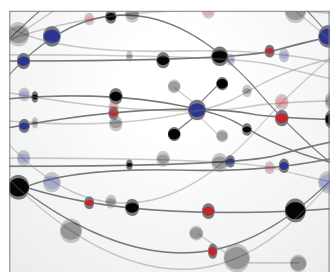

\section{The Scientific} World Journal
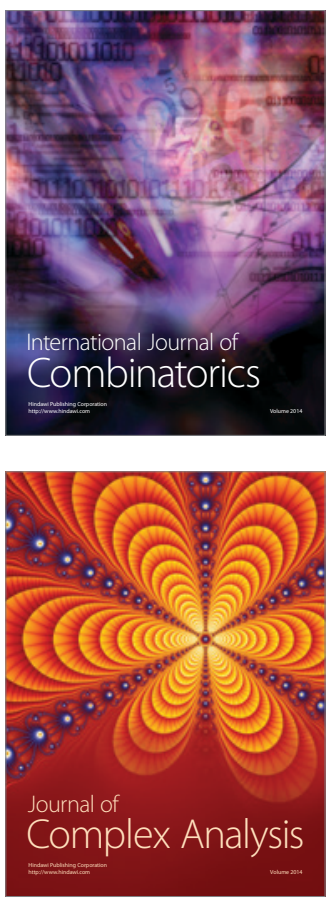

International Journal of

Mathematics and

Mathematical

Sciences
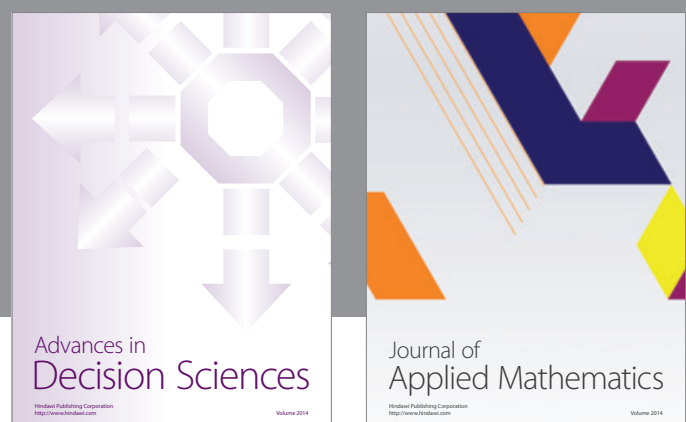

Journal of

Applied Mathematics
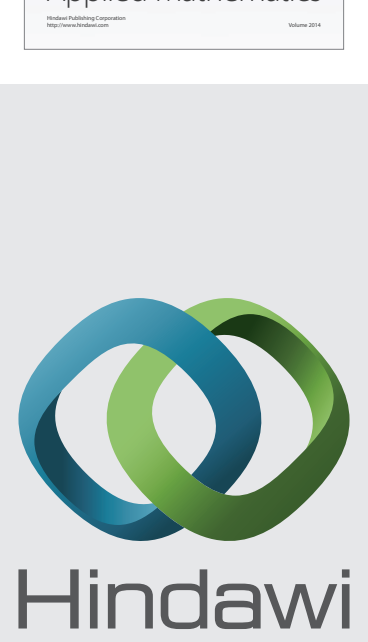

Submit your manuscripts at http://www.hindawi.com
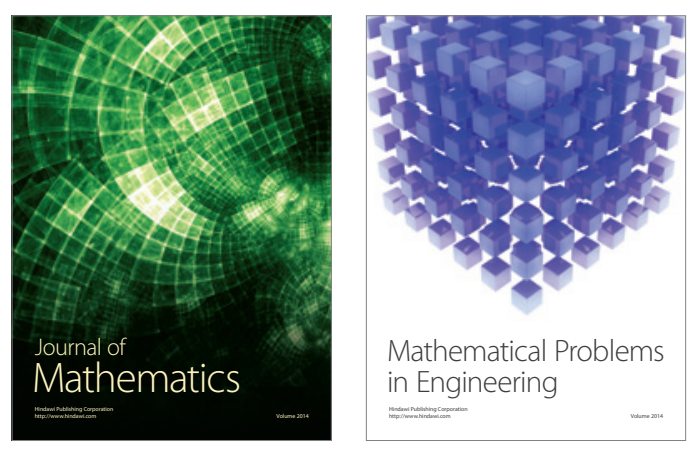

Mathematical Problems in Engineering
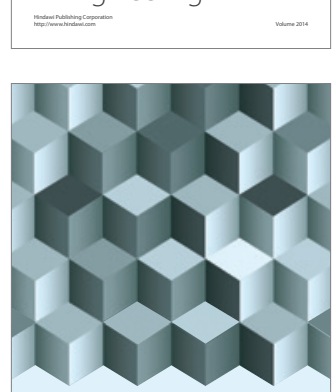

Journal of

Function Spaces
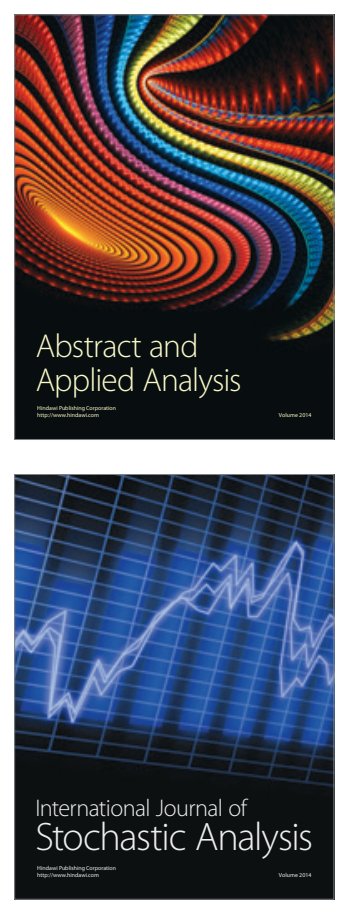

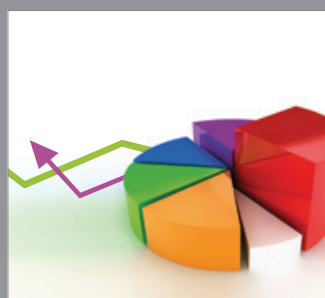

ournal of

Probability and Statistics

Promensencen
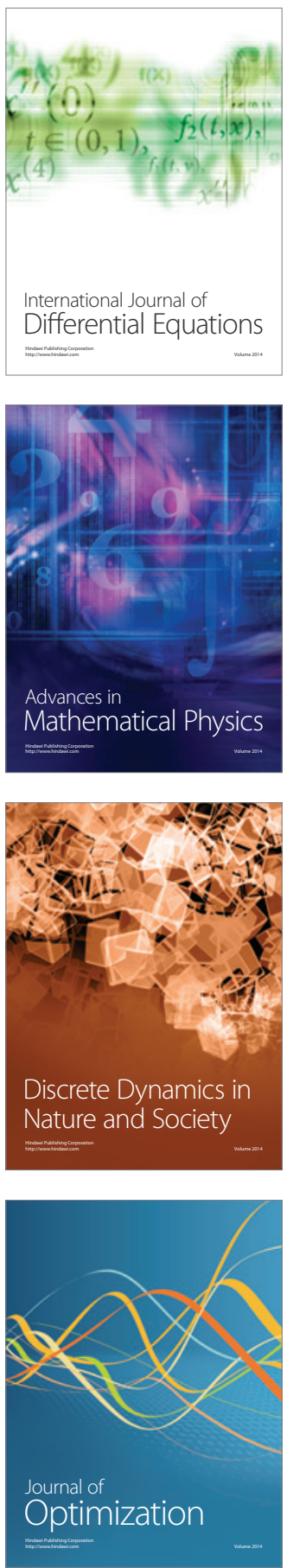